finkResearch Article submitted to Virus Evolution:

\title{
Differential Disease Severity and Whole Genome Sequence Analysis for Human Influenza A/H1N1pdm Virus in 2015-2016 Influenza Season
}

Hsuan Liu ${ }^{1, \#}$, Yu-Nong Gong ${ }^{2,3, \#}$, Kathryn Shaw-Saliba ${ }^{1,4}$, Thomas Mehoke ${ }^{5}$, Jared Evans $^{5}$, Zhen-Ying Liu ${ }^{6}$, Mitra Lewis ${ }^{4}$, Lauren Sauer ${ }^{4}$, Peter Thielen ${ }^{5}$, Richard Rothman ${ }^{4}$, Kuan-Fu Chen $6,7,8, \dagger$, Andrew Pekosz ${ }^{1,4,9, \dagger}$

${ }^{1}$ W. Harry Feinstone Department of Molecular Microbiology and Immunology, The Johns Hopkins Bloomberg School of Public Health, Baltimore, Maryland 21205 USA

${ }^{2}$ Research Center for Emerging Viral Infections, College of Medicine, Chang Gung University, Taoyuan, Taiwan

${ }^{3}$ Department of Laboratory Medicine, Linkou Chang Gung Memorial Hospital, Taoyuan, Taiwan

${ }^{4}$ Department of Emergency Medicine, Johns Hopkins University School of Medicine, Baltimore, Maryland 21205 USA

${ }^{5}$ Research and Exploratory Development Department, Johns Hopkins Applied Physics Laboratory, Laurel, Maryland 20723 USA

${ }^{6}$ Department of Emergency Medicine, Chang Gung Memorial Hospital, Keelung, Taiwan ${ }^{7}$ Clinical Informatics and Medical Statistics Research Center, Chang Gung University, Taoyuan, Taiwan

${ }^{8}$ Community Medicine Research Center, Chang Gung Memorial Hospital, Keelung, Taiwan

${ }^{9}$ Department of Environmental Health and Engineering, The Johns Hopkins Bloomberg School of Public Health, Baltimore, Maryland 21205 USA

\# These authors contributed equally to the manuscript.

† Correspondence should be addressed to Kuan-Fu Chen, Department of Emergency Medicine, Chang Gung Memorial Hospital, Keelung, Taiwan, kfchen@cgmh.org.tw, or Andrew Pekosz, W. Harry Feinstone Department of Molecular Microbiology and Immunology, Johns Hopkins University Bloomberg School of Public Health, Baltimore, Maryland, 21205, USA, Phone: +1-410-502-9306, Fax: +1-410-955-0105, email: apekosz1@jhu.edu

Abstract: $232 / 350$

Main Text: $3571 / 10000$ 


\section{Abstract:}

42 During the 2015-16 winter, the US experienced a relatively mild influenza season compared to Taiwan which had a higher number of total and severe cases. While

$44 \quad \mathrm{H} 1 \mathrm{~N} 1 \mathrm{pdm}$ viruses dominated global surveillance efforts that season, the global distribution of genetic variants and their contributions to disease severity have not been investigated. Samples collected from influenza A positive patients by the Johns Hopkins

47 Center of Excellence for Influenza Research and Surveillance (JH-CEIRS) active surveillance in the emergency rooms in Baltimore, Maryland, USA and northern Taiwan between November 2015 and April 2016, were processed for influenza A virus whole genome sequencing. In Baltimore, the majority of the viruses were the H1N1pdm clade 6B.1 and no H1N1pdm clade 6B.2 viruses were detected. In northern Taiwan, more

52 than half of the $\mathrm{H} 1 \mathrm{~N} 1 \mathrm{pdm}$ viruses were clade $6 \mathrm{~B} .1$ and $38 \%$ were clade $6 \mathrm{~B} .2$,

53 consistent with the global observation that most 6B.2 viruses circulated in Asia and not

54 North America. Whole virus genome sequence analysis identified two genetic

55 subgroups present in each of the 6B.1 and 6B.2 clades and one 6B.1 intraclade

56 reassortant virus. Clinical data showed 6B.2 patients had more disease symptoms

57 including higher crude and inverse probability weighted odds of pneumonia than 6B.1

58 patients, suggesting 6B.2 circulation may contribute to the severe flu season in Taiwan.

59 Local surveillance efforts linking H1N1pdm virus sequences to patient clinical and 60 demographic data improve our understanding of influenza circulation and disease 61 potential. 
bioRxiv preprint doi: https://doi.org/10.1101/2020.02.20.957068; this version posted October 3, 2020. The copyright holder for this preprint (which was not certified by peer review) is the author/funder, who has granted bioRxiv a license to display the preprint in perpetuity. It is made available under aCC-BY-NC-ND 4.0 International license.

Liu et al.

63 Key words: influenza, H1N1pdm, whole genome sequencing, disease severity, Taiwan,

\section{Baltimore}

65 
Liu et al. $\quad 4$

Introduction:

67 The 2009 pandemic H1N1 Influenza A virus (H1N1pdm) has supplanted the previous

human $\mathrm{H} 1 \mathrm{~N} 1$ viruses to become the seasonal human $\mathrm{H} 1 \mathrm{~N} 1$ virus. In the 2015-16

69 influenza season, the northern hemisphere experienced its second global $\mathrm{H} 1 \mathrm{~N} 1 \mathrm{pdm}-$ dominant year since 2009. The 2015-16 Influenza activity in the US was considered

71 moderate, with lower numbers of total cases and a later peak epidemic when compared with the previous three influenza seasons [1]. However, in Taiwan, the 2015-16 season was much more serious [2], with an earlier start and higher numbers of total as well as severe, influenza cases compared to the prior influenza season 2014-15 [3].

Influenza A viruses (IAV) are subdivided into subtypes based on the antibody response to the viral glycoproteins, hemagglutinin (HA) and neuraminidase (NA). Viruses are further divided into genetic clades based on HA sequences in order to monitor for mutations that might lead to antigenic drift. The 2015-16 northern hemisphere vaccine strain of H1N1pdm, A/California/7/2009, is defined as H1 clade 1. Prior to the 2015-16 season, $\mathrm{H} 1 \mathrm{~N} 1 \mathrm{pdm}$ was dominated by clade $6 \mathrm{~B}$. The global rise of $\mathrm{H} 1$ clade $6 \mathrm{~B} .1$ carrying amino acid changes S84N, S162N and I216T from clade 6B was detected in human surveillance efforts starting in June 2015 and is now the dominant H1N1 clade. emerged in July 2015, peaked in January 2016, were found primarily in Asia and disappeared from surveillance efforts at the end of 2016 [4, 5]. The majority of $6 \mathrm{~B} .1$ and 
89 although antigenic difference between egg-adapted and circulating virus strains may

90 have contributed to increased numbers of cases [8].

92 The genome of IAV consist of 8 segments of negative-sense RNA. In addition to HA mutations, mutations in the other 7 viral gene segments also result in genetic variants which can alter virus fitness, subvert pre-existing immunity and/or reduce vaccine effectiveness. Any of these changes could potentially alter disease severity or total number of cases [9]. Next-generation sequencing provides a sensitive and rapid method

97 for sequencing of viruses directly from human specimens without any need for virus isolation or culture - both of which are known to select for viruses bearing additional 99 mutations [10].

Most current global influenza surveillance systems lack connections between viral sequences and detailed patient demographic and clinical data. The Johns Hopkins Center of Excellence for Influenza Research and Surveillance (JH-CEIRS) has been actively performing human influenza surveillance since 2014. Surveillance efforts involve the connection of viral genome data and isolates with patient demographic and

106 clinical data to gain more insights into how virus genetic variation can affect clinical

107 disease. We hypothesized that sequence differences in influenza viruses could be 108 associated with distinct clinical outcomes. This study involved concurrent, active 109 influenza surveillance during the $2015-16$ season in Baltimore, USA and northern 110 Taiwan to compare and contrast differential epidemics in the same year between the 
111 two geographic regions and to analyze possible disease severity with influenza viral

112 genotypes.

114 Materials and Methods:

Active surveillance enrollment and sample collection

116 Institutional Review Boards at the Johns Hopkins University School of Medicine and

117 Chang Gung Memorial Hospital provided ethical approval of the study (IRB00052743).

118 From November 2015 to April 2016, active surveillance was performed in the adult emergency departments of the Johns Hopkins Medical Institutions (JHMI) at the East Baltimore and Bayview campuses in Baltimore, Maryland, USA and Chang Gung

121 Memorial Hospitals (CGMH) in the northern Taiwan metropolitan area, including

122 hospitals in the Taipei, Linkou, and Keelung Branches. Influenza-like illness was defined

123 as documented or reported $\geq 38^{\circ} \mathrm{C}$ fever and any of one of three respiratory symptoms

124 including cough, headache and/or sore throat within the past 7 days. Exclusion criteria

125 included subjects who are unable to speak and understand English (in US) or Mandarin

126 (in Taiwan), unable to provide written informed consent, currently incarcerated, or

127 previously enrolled in the study during the same influenza season. Patients were

128 approached by trained clinical coordinators who obtained written, informed consent

129 before collected specimens and demographic and clinical data using a standard

130 questionnaire. Data was confirmed by examination of the patient's electronic health

131 record. All data was de-identified and stored in a secure REDCap database [11, 12].

\section{Diagnosis and subtyping}


134 Nasopharyngeal swabs or nasal washes of patients collected at the time of enrollment were tested for influenza A virus infection using the Cepheid Xpert Flu/RSV test (Cepheid, Sunnyvale, CA). Samples that were influenza A virus positive were further subtyped by qRT-PCR with specific $\mathrm{H} 1$ or $\mathrm{H} 3$ primers and probes according to USA Centers for Disease Control and Prevention (CDC) protocols. Only H1N1pdm positive samples were moved on to whole genome sequencing (WGS) analysis.

\section{Whole genome sequencing}

142 Viral RNA was isolated from the influenza A positive clinical samples using the MagMax

143 Viral RNA isolation reagent (Thermo Fisher) on an Eppendorf epMotion 5073 liquid

144 handling workstation. Samples were processed for WGS using multi-segment PCR [10]

145 and then prepared for deep sequencing using the Nextera XT library preparation

146 reagent (Illumina). Samples were sequenced on the Illumina NextSeq platform. Raw

147 paired-end data were first processed through Trim galore!

148 (https://www.bioinformatics.babraham.ac.uk/projects/trim galore/) with a quality score of 14930 and an adapter sequence of CTGTCTCTTATACACATCT, only retaining pairs that 150 both passed through this quality control step. The quality-trimmed reads were then 151 aligned to the influenza vaccine strain reference sequence using bowtie2 (version 2.1.0) 152 [13] with the '--very-sensitive-local' option and converted to sorted BAM files using 153 samtools [14]. Only sequences of all 8 gene segments passed quality controls were 154 included in the study. The sequences were submitted to NCBI GenBank database and 155 accession numbers are listed in the Table S1. 


\section{Phylogenetics and sequence analysis}

73 H1N1pdm clinical samples which yielded sufficient WGS coverage of the IAV genome were included in sequence analysis. In addition to these genomes, we retrieved two reference genomes of vaccine strains from the Global Initiative on Sharing All Influenza Data (GISAID, https://www.gisaid.org/) for further analyses. Nucleotide sequences of the longest open reading frames (ORFs) in each gene segment were used to build phylogenetic trees, including PB2, PB1, PA, HA, NP, NA, M1, and NS1, as well as, their alternative splicing (M2 and NS2) and ribosomal frameshift (PA-X) products. Nucleotide sequences of these ORFs (except for PA-X) in each strain were

171 model in TreeTime [16] after building ML trees. Clade 6B, 6B.1, and 6B.2 were defined by the rules of HA amino acid mutations of global surveillance groups from nextflu [4].

173 Phylogenetic groups in other gene segments were based on their tree branches to

174 separate annotated clades (e.g., 6B.1 and 6B.2). Inconsistent positioning of strains, or

175 clade groups on individual trees was used to identify influenza reassortment. Individual 176 gene segment clades were annotated and visualized using the ggtree R package [17]. 
180 tool [18] under the default conditions. Moreover, we retrieved all NP sequences

$181(n=20,477)$ of H1N1pdm isolated after the year of 2009 (inclusive) from GISAID as of

182 January 2020 to investigate the population of viruses encoding an upstream translation

183 initiation codon (AUG) in the NP gene segment. 20,470 NP sequences were used for

184 further analyses, after aligning and removing 7 sequences with unexpected gaps.

\section{Glycosylation prediction}

187 Potential N-linked glycosylation sites of the 6B.1 and 6B.2 HA and NA proteins were 188 predicted by the NetNGlyc 1.0 server (http://www.cbs.dtu.dk/services/NetNGlyc).

\section{Statistical analysis of demographic and clinical data}

To identify a contribution of virus clades and genetic subgroups to clinical symptoms,

we applied Chi-square or Fisher's exact tests for categorical variables and the rank-sum test for continuous variables when appropriate. The two-tailed statistical significance was set at $p$-value $<0.05$. When clinical or demographic category reached statistical significance in a univariate analysis, confounding effects were then analyzed. In addition to adjusting for possible confounding effects between demographics, disease severity

197 and clinical outcomes, a propensity score-based weighting was applied. Briefly, we 198 applied those potential confounders to generate the propensity (or probability) of being 199 infected with different subgroups of H1N1pdm viruses by using machine learning based modeling methods [19]. By using the inverse probability of treatment weighting (IPTW)

201 method, patients with higher propensity will have lower weights in the final logistic 
203 population where the weighted virus subgroups have similar distribution across these

204

205

206

207

208

209

210

211

212

213

214

215

216

217

218

219

220

221

222

223

224

225

confounders. In one simulation model, the propensity score-based weighting methods

could adjust for most of the confounding effects [20]. Odds ratios of unadjusted and

IPTW were calculated using logistic regression analysis.

\section{Results:}

H1N1pdm clade 6B.1 predominantly circulated in Baltimore and clade 6B.1 and

\section{B.2 co-circulated in northern Taiwan}

From November 2015 to April 2016, 261 and 256 symptomatic patients were enrolled in the study from emergency departments in JHMI (Baltimore) and CGMH (northern

Taiwan). IAV was found in 32.6\% (85/261) of symptomatic patients from Baltimore and $57.8 \%$ (148/256) from northern Taiwan. About half of the IAV-positive samples were further subtyped, and the demographics and comorbidities were found to be similar. In $97.5 \%(39 / 40)$ of samples from Baltimore and $90.1 \%(64 / 71)$ of samples from northern

Taiwan H1N1 was the influenza A virus subtype. The epidemic curves of the two surveillance sites were different, with influenza A virus activity starting in January and peaking in mid-March in Baltimore while IAV activity in northern Taiwan started in November and peaked in late January/early February, with peak activity higher than in Baltimore (Fink et al., MedRxiv).

Seventy-three of the $103 \mathrm{H} 1 \mathrm{~N} 1 \mathrm{pdm}$ clinical samples yielded sufficient sequence coverage of the IAV genome. Virus samples were initially analyzed based on HA segment sequences (Figure 1). In Baltimore, 91.7\% (22/24) of viruses were clade 6B.1, 
226 two were clade 6B, and no 6B.2 viruses were identified. The northern Taiwan samples

227 consisted of $57.1 \%$ (28/49) clade 6B.1, 38.8\% (19/49) clade 6B.2 and 4.1\% (2/49) clade

228 6B viruses. The difference in clade circulation is consistent with global influenza

229 surveillance that $\mathrm{H} 1 \mathrm{~N} 1 \mathrm{pdm}$ 6B.1 and 6B.2 were dominant in the 2015-16 season and 230 most 6B.2 viruses were found in Asia [5, 21].

\section{Identification of four distinct genetic subgroups of H1N1pdm}

233 To better understand the genetic diversity in the identified HA clades, we performed a phylogenetic analysis using concatenated gene segments (Figure 2) and individual ORF trees (Figure S1 and Figure 2). Branch tips of the 10 ORFs from the other seven gene segments were colored by the HA clade of each sample and defining amino acid

237 mutations were noted (Figure S1). For the most part, the sequences of other ORFs 238 clustered together consistent with their HA clade (Figure 2). Amino acid differences 239 between clades 6B.1 and 6B.2 are listed for HA (Table S2), NA (Table S3) and the 240 other ORFs (Table S4).

242 Additional amino acid mutations were identified to differentiate viruses into four distinct

243 virus genetic subgroups (Figure 2). The 6B.1 viruses were separated into two groups, a

244 6B.1 parental and a 6B.1 variant defined by unique amino acid changes in NA and NS2

245 (Table S3 and S4). All Baltimore 6B.1 viruses were 6B.1 parental. In northern Taiwan, 246 only 4 were 6B.1 parental, with the remaining clade 6B.1 viruses (23/27) being 6B.1

247 variant. The 6B.2 viruses were also separated into two genetic subgroups - variant-1 
and variant-2 - that are differentiated by mutations in HA, NA, M1 and M2 (Table S2, S3 and S4).

\section{Dynamic virus evolution of gene segments and possible reassortment}

252 All segments in two of the four 6B viruses, A/EastBaltimore/0103/2016 and viruses were evolving toward either the clade 6B.1 or clade 6B.2.

There was also evidence of intraclade reassortment in one northern Taiwan 6B.1 intraclade reassortant virus.

\section{HA and NA N-linked glycosylation}

269 Eight N-linked glycosylation sites on the HA of A/California/7/2009 were previously 
271 sequence predicts an additional N-linked glycosylation site at 162-164 (N-Q-S) on the

272 Sa antigenic site of the head domain due to a S to N mutation at HA-162 (Table S2)

273 [23]. The NA from the A/California/7/2009 vaccine strain has 8 potential $\mathrm{N}$-linked

274 glycosylation sites and 6 sites were experimentally confirmed [22]. Both 6B.1 and 6B.2

275 viruses gain one additional predicted N-linked glycosylation site at 42-44 (N-Q-S) of the

276 NA stalk and lose one at 386-388 (N-F-S) which lies on the lateral surface of the NA

277 head. In addition, 68.4\% (13/19) of 6B.2 viruses with NA-S52R mutation lose one

278 glycosylation site at 50-52 (N-Q-S) on the NA stalk (Table S3).

279

\section{Antiviral resistance mutations}

281 All sequences contained the amantadine resistance mutation M2-S31N which is found

282 in the $\mathrm{A} / \mathrm{Cal} / 07 / 2009$ virus. One 6B.2 variant-2 sample from northern Taiwan,

A/Linkou/0029/2015, contained the NA-H275Y, the most common mutation associated with oseltamivir resistance in N1 NA.

An upstream AUG codon lengthens the nucleoprotein (NP) of H1N1pdm

287 The NP gene segments of all samples in the study have a new, in frame AUG start

288 codon 18 nucleotides upstream of the usual start codon of NP proteins of IAV including

289 human seasonal $\mathrm{H} 1 \mathrm{~N} 1$ and $\mathrm{H} 3 \mathrm{~N} 2$ [24, 25], adding 6 amino acids to the amino terminus

290 of the protein with clade 6B.1 (M-S-D-I-E-A) and 6B.2 viruses (M-S-D-I-E-V) differing at

291 amino acid six. This start codon was shown to be utilized and the resulting NP protein

292 capable of supporting viral polymerase activity $[26,27]$. The presence of an upstream

293 NP start codon and the amino acid difference at position 6 between 6B.1 and 6B.2 were 
Liu et al. $\quad 14$

294 also present in approximately $70 \%$ of GISAID sequences $(n=20,470)$ of $\mathrm{H} 1 \mathrm{~N} 1 \mathrm{pdm}$ NP

295 since 2009 with an Alanine (A) dominating at position 6.

Patients infected with clade 6B.2 had increased symptoms of severe influenza

To determine whether viral genotype contributes to symptoms and disease severity, the demographic and clinical data from patients infected with clade 6B.1 or 6B.2 viruses were first compared by univariate analyses (Table 1). Patients infected with 6B.2 were

301 more likely to be older, female, Asian, and unvaccinated. These patients had a higher prevalence of runny nose and diarrhea and had increased symptoms of severe influenza, including increased likelihood of oxygen supplementation, longer in-hospital stay, and pneumonia diagnosis (by radiological findings). Interestingly, patients infected with clade 6B.1 viruses had a lower number of co-morbidities associated with severe influenza, suggesting a healthier population was being infected with those viruses.

We further utilized a logistic regression analysis to estimate the propensity of being infected by 6B.2, using potential confounders such as age, gender, comorbidities, obesity, vaccine status, human exposure and travel history (area under the ROC curve: 
317 of wheezing, sinus pain, and nausea/vomiting, along with the expected difference in race and ethnicity stemming from the identification of the 6B.1 variant only in northern Taiwan (Table 3). When patients infected with the 6B.2 variant-1 and variant-2 viruses were compared there were no obvious difference in symptoms (Table 4), however, the small sample sizes in the 6B.2 subgroups precluded a definitive characterization of

322 demographic and clinical data between these genotypes. Overall, our data suggested clade 6B.2 circulation in northern Taiwan likely played a significant role in differentiating

324 the influenza epidemics between Baltimore and northern Taiwan in the season.

\section{Discussion:}

327 Four distinct genetic subgroups of $\mathrm{H} 1 \mathrm{~N} 1 \mathrm{pdm}$ viruses were circulating in Baltimore and northern Taiwan during the 2015-16 flu season. While the 6B.1 clade was circulating at both sites, some 6B.1 viruses in northern Taiwan had additional mutations which defined a genetic subgroup we called 6B.1 variant. The two 6B.1 subgroups of viruses did not differ drastically with respect to disease severity. The 6B.2 clade viruses were detected in northern Taiwan but not in Baltimore and consisted of two distinct genetic subgroups. Patients infected with 6B.2 virus showed more and stronger disease symptoms by both uni- and multivariate analysis. This difference in $\mathrm{H} 1 \mathrm{~N} 1 \mathrm{pdm}$ clade circulation may have contributed to the more severe influenza season experienced in northern Taiwan compared to Baltimore in 2015-16. After adjusting and weighting for age, gender, comorbidities, obesity, vaccine status, human exposure and travel history, people infected with 6B.2 still had a higher odds ratio of pneumonia (Table 2). The data

339 indicate that infection with clade 6B.2 viruses is associated with a higher disease 
severity at our surveillance sites independent of patient demographics and comorbidities. The presence of the 6B.2 clade in addition to low preexisting immunity to circulating H1N1 in northern Taiwan ( reference Fink, 2020 medrxiv) may be the most likely reasons explaining the different numbers and severity of influenza cases between Baltimore and northern Taiwan. The only report for symptoms of $6 \mathrm{~B} .1$ compared to $6 \mathrm{~B} .2$ infection was seen in Israel, where infection with 6B.2 virus was associated with more vomiting and nausea compared to 6B.1-infected patients [28]. In our surveillance, we did see a higher percentage of 6B.2 than 6B.1 patients with vomiting and nausea, but this did not reach statistical significance (Table 1).

The H1N1pdm 6B.1 and 6B.2 viruses dominated the 2015-16 season in the Northern Hemisphere. However, numbers of cases and disease severity differed between geographic regions. The 2015-16 H1N1pdm season in the US and Canada were moderate compared to the prior season $[1,6]$. On the other hand, influenza surveillance in Russia, UK, eastern Europe, the Middle East and Asia (including Taiwan) had increasing numbers of severe cases $[3,7,29-33]$. Reports also showed H1N1pdm impacted the southern hemisphere in the 2016 season, in particular, Brazil and Reunion Island [34-36].

Serological surveillance indicated that the circulating H1N1pdm strains in 2015-16 were antigenically similar to the vaccine A/California/7/2009 in the US, UK and many other countries $[1,6,7,32,37,38]$. This suggests that the HA amino acid differences between the circulating $6 \mathrm{~B} .1$ or $6 \mathrm{~B} .2$ viruses and the vaccine strain were likely not able 
363 to explain differential epidemics in different regions of the year even though vaccination coverage might differ. Whether mutations in the other 7 viral genes contributed to the variable disease severity reported in 2015-16 has not been studied carefully. Some mutations in NA and M1 occurred at sites previously associated with altered virus transmission [39, 40], but our initial experiments on human nasal epithelial cell cultures did not detect any replication differences between clade 6B.1 and 6B.2 viruses ( reference Fink, 2020 medrxiv).

371 We identified one 6B.2 patient in northern Taiwan had NA-H275Y mutation, which is

372 associated with NA drug resistance. In another study of 2015-16 H1N1pdm in Taiwan,

373 one clinical isolate with NA-H275Y was resistant to oseltamivir [41]. The data suggest

374 sporadic identification of oseltamivir resistant H1N1pdm viruses in 2015-16 in Taiwan.

376 The clade 6B.1 parental genetic subgroup continued to circulate after 2015-16, and

377 evolved into several 6B.1A subclades through August 2020. It is not clear what drove

378 the extinction of $6 \mathrm{~B} .2$ but it is possible that clade $6 \mathrm{~B} .1$ viruses were better adapted to

379 infect and spread in humans. It has been suggested that glycosylation is important for

380 influenza A virus adaptation in humans [42] and gaining a potential glycosylation site on

381 the HA head at residue 162 may have given clade 6B.1 viruses an evolutionary

382 advantage. Clade 6B.1 viruses also encode an NS1-E125D mutation which increases

383 NS1 interactions with cellular cleavage and polyadenylation factor 30 (CPSF30) [43].

384 The NS1-E125D mutation of 6B.1 viruses has been suggested to be an important 385 marker for virus adaption to humans [7]. 
387 Our study is limited in several ways. The different race distributions between our surveillance sites make it difficult to adjust for in this and any study conducted across different geographic regions. The identification of 4 genetic subgroups reduced the power of our study to detect demographic and clinical differences in our populations.

391 Increasing surveillance and whole genome sequencing efforts could dramatically 392 improve the ability to identify novel virus variants that are causing altered disease in 393 humans. Given these limitations, it is still clear that there is significant H1N1 genetic diversity across the two surveillance sites and this genetic diversity contributes to the 395 differing number of influenza cases and disease severity. Influenza is a global disease, 396 but surveillance at the local level is needed to fully understand virus circulation and 397 disease potential.

\section{Conflicts of interests}

400 The authors declare no conflict of interest.

401

\section{Author contributions}

403 H.L., R.R., K.F.C., and A.P. conceived of the experimental questions; Y.N.G. performed 404 phylogenetic tree analyses; K.S.S., and K.F.C. ran demographic and clinical analyses; 405 T.M., J.E., and P.T. sequenced samples and cleaned the raw data; Z.Y.L., M.L., and 406 L.S. oversaw enrollment and collection of samples from patients; H.L., Y.N.G., K.S.S., 407 and K.F.C. analyzed data; H.L., and A.P. wrote the manuscript; all authors edited and 408 reviewed the manuscript prior to submission. 


\section{$410 \quad$ Funding}

411 This work was supported by the NIH/NIAID Center of Excellence in Influenza Research

412 and Surveillance contract HHS N2772201400007C (R.R., K.F.C., and A.P.), the Richard

413 Eliasberg Family Foundation and the Research Center for Emerging Viral Infections

414 from The Featured Areas Research Center Program within the framework of the Higher

415 Education Sprout Project by the Ministry of Education (MOE) in Taiwan and the Ministry

416 of Science and Technology (MOST), Taiwan MOST 109-2634-F-182-001 (Y.N.G.).

\section{Acknowledgements}

419 The authors thank the patients who enrolled and participated in the JH-CEIRS

420 surveillance study. We are grateful for the efforts of the clinical coordination team at

421 JHMI who collected samples. We thank members of the Pekosz lab for feedback on this

422 work.

423

\section{References:}

1. Davlin, S.L., et al., Influenza Activity - United States, 2015-16 Season and Composition of the 2016-17 Influenza Vaccine. MMWR Morb Mortal Wkly Rep, 2016. 65(22): p. 567-75.

428 2. Gong, Y.N., et al., Centennial review of influenza in Taiwan. Biomed J, 2018. 41(4): p. 234-241.

429 3. Taiwan_CDC. Taiwan Influenza Express 2015-16 Flu Season 2016 Week 20. 2016; Available from: https://www.cdc.gov.tw/En/File/Get/VCNkIvtTOiuP1vo1gJG89A.

4314 Neher, R.A. and T. Bedford, nextflu: real-time tracking of seasonal influenza virus evolution in humans. Bioinformatics, 2015. 31(21): p. 3546-8.

5. Bedford, T. and R. Neher. Seasonal influenza circulation patterns and projections for 2017-2018. 2017; Available from: https://nextflu.org/reports/feb-2017/. 
bioRxiv preprint doi: https://doi.org/10.1101/2020.02.20.957068; this version posted October 3, 2020. The copyright holder for this preprint (which was not certified by peer review) is the author/funder, who has granted bioRxiv a license to display the preprint in perpetuity. It is made available under aCC-BY-NC-ND 4.0 International license.

Liu et al. $\quad 20$

7. Komissarov, A., et al., Rapid spread of influenza A(H1N1)pdm09 viruses with a new set of specific mutations in the internal genes in the beginning of 2015/2016 epidemic season in Moscow and Saint Petersburg (Russian Federation). Influenza Other Respir Viruses, 2016. 10(4): p. 247-53.

8. Garretson, T.A., et al., Identification of human vaccinees that possess antibodies targeting the egg-adapted hemagglutinin receptor binding site of an H1N1 influenza vaccine strain. Vaccine, 2018. 36(28): p. 4095-4101.

9. $\quad$ Bouvier, N.M. and P. Palese, The biology of influenza viruses. Vaccine, 2008. 26 Suppl 4: p. D4953.

10. Zhou, B., et al., Single-reaction genomic amplification accelerates sequencing and vaccine production for classical and Swine origin human influenza a viruses. J Virol, 2009. 83(19): p. 10309-13.

11. Harris, P.A., et al., Research electronic data capture (REDCap)--a metadata-driven methodology and workflow process for providing translational research informatics support. J Biomed Inform, 2009. 42(2): p. 377-81.

12. Harris, P.A., et al., The REDCap consortium: Building an international community of software platform partners. J Biomed Inform, 2019. 95: p. 103208.

13. Langmead, B. and S.L. Salzberg, Fast gapped-read alignment with Bowtie 2. Nat Methods, 2012. 9(4): p. 357-9.

14. Li, H., et al., The Sequence Alignment/Map format and SAMtools. Bioinformatics, 2009. 25(16): p. 2078-9.

15. Stamatakis, A., RAXML version 8: a tool for phylogenetic analysis and post-analysis of large phylogenies. Bioinformatics, 2014. 30(9): p. 1312-3.

16. Sagulenko, P., V. Puller, and R.A. Neher, TreeTime: Maximum-likelihood phylodynamic analysis. Virus Evol, 2018. 4(1): p. vex042.

17. Yu, G., et al., ggtree: an r package for visualization and annotation of phylogenetic trees with their covariates and other associated data. Methods Ecol Evol, 2017. 8(1): p. 28-36.

18. Katoh, K. and D.M. Standley, MAFFT multiple sequence alignment software version 7: improvements in performance and usability. Mol Biol Evol, 2013. 30(4): p. 772-80.

19. Lee, B.K., J. Lessler, and E.A. Stuart, Improving propensity score weighting using machine learning. Stat Med, 2010. 29(3): p. 337-46.

20. Stuart, E.A., Matching methods for causal inference: A review and a look forward. Stat Sci, 2010. 25(1): p. 1-21.

21. World_Health_Organization, Recommended composition of influenza virus vaccines for use in the 2016-2017 northern hemisphere influenza season. Wkly Epidemiol Rec, 2016. 91(10): p. 12132.

22. She, Y.M., et al., Topological N-glycosylation and site-specific $N$-glycan sulfation of influenza proteins in the highly expressed H1N1 candidate vaccines. Sci Rep, 2017. 7(1): p. 10232.

23. Altman, M.O., et al., Human Influenza A Virus Hemagglutinin Glycan Evolution Follows a Temporal Pattern to a Glycan Limit. mBio, 2019. 10(2).

24. Reid, A.H., et al., Novel origin of the 1918 pandemic influenza virus nucleoprotein gene. J Virol, 2004. 78(22): p. 12462-70.

25. Gorman, O.T., et al., Evolution of influenza A virus nucleoprotein genes: implications for the origins of H1N1 human and classical swine viruses. J Virol, 1991. 65(7): p. 3704-14.

26. Wise, H.M., et al., An alternative AUG codon in segment 5 of the 2009 pandemic influenza $A$ virus is a swine-derived virulence motif. bioRxiv, 2019: p. 738427.

27. Wanitchang, A., et al., Atypical characteristics of nucleoprotein of pandemic influenza virus H1N1 and their roles in reassortment restriction. Arch Virol, 2011. 156(6): p. 1031-40. 
bioRxiv preprint doi: https://doi.org/10.1101/2020.02.20.957068; this version posted October 3, 2020. The copyright holder for this preprint (which was not certified by peer review) is the author/funder, who has granted bioRxiv a license to display the preprint in perpetuity. It is made available under aCC-BY-NC-ND 4.0 International license.

Liu et al.

28. Friedman, N., et al., A(H1N1)pdm09 influenza infection: vaccine inefficiency. Oncotarget, 2017. 8(20): p. 32856-32863.

29. Tjon-Kon-Fat, R., et al., The potential risks and impact of the start of the 2015-2016 influenza season in the WHO European Region: a rapid risk assessment. Influenza Other Respir Viruses, 2016. 10(4): p. 236-246.

30. Newitt, S., et al., Rapid risk assessment during the early weeks of the 2015-2016 influenza season in Ukraine. Influenza Other Respir Viruses, 2018. 12(2): p. 241-249.

31. Public_Health_England_(PHE). Summary of UK surveillance of influenza and other seasonal respiratory illnesses 26 May 2016 - Week 21 report (up to week 20 data). PHE weekly national influenza report. 2016; Available from: https://assets.publishing.service.gov.uk/government/uploads/system/uploads/attachment dat a/file/681012/Weekly national influenza report week 21 2016.pdf.

32. Pebody, R., et al., Effectiveness of seasonal influenza vaccine in preventing laboratory-confirmed influenza in primary care in the United Kingdom: 2015/16 mid-season results. Euro Surveill, 2016. 21(13).

33. National_Institute_of_Infectious_Diseases_Japan, Influenza 2015/16 season, Japan. Infectious Agents Surveillance Report, 2016. 37(11): p. 211-213.

34. Santos, K.C., et al., Molecular epidemiology of influenza A(H1N1)PDM09 hemagglutinin gene circulating in Sao Paulo State, Brazil: 2016 anticipated influenza season. Rev Inst Med Trop Sao Paulo, 2017. 59: p. e9.

35. Cardoso, A.M., et al., Investigation of an outbreak of acute respiratory disease in an indigenous village in Brazil: Contribution of Influenza $A(H 1 N 1) p d m 09$ and human respiratory syncytial viruses. PLoS One, 2019. 14(7): p. e0218925.

36. Filleul, L., et al., A major impact of the influenza seasonal epidemic on intensive care units, Reunion, April to August 2016. Euro Surveill, 2016. 21(47).

37. Broberg, E., et al., Predominance of influenza A(H1N1)pdm09 virus genetic subclade $6 B .1$ and influenza B/Victoria lineage viruses at the start of the 2015/16 influenza season in Europe. Euro Surveill, 2016. 21(13).

38. Korsun, N., et al., Antigenic and genetic characterization of influenza viruses circulating in Bulgaria during the 2015/2016 season. Infect Genet Evol, 2017. 49: p. 241-250.

39. Lv, J., et al., Amino acid substitutions in the neuraminidase protein of an H9N2 avian influenza virus affect its airborne transmission in chickens. Vet Res, 2015. 46: p. 44.

40. Bialas, K.M., E.A. Desmet, and T. Takimoto, Specific residues in the 2009 H1N1 swine-origin influenza matrix protein influence virion morphology and efficiency of viral spread in vitro. PLoS One, 2012. 7(11): p. e50595.

41. Gong, Y.N., et al., Population dynamics at neuraminidase position 151 of influenza $A$ (H1N1)pdm09 virus in clinical specimens. J Gen Virol, 2019. 100(5): p. 752-759.

42. Tate, M.D., et al., Playing hide and seek: how glycosylation of the influenza virus hemagglutinin can modulate the immune response to infection. Viruses, 2014. 6(3): p. 1294-316.

43. Hale, B.G., et al., Inefficient control of host gene expression by the 2009 pandemic H1N1 influenza A virus NS1 protein. J Virol, 2010. 84(14): p. 6909-22. 
527 Figure Legends.

528 Figure 1. H1N1pdm clade 6B.1 and clade 6B.2 circulated in Baltimore and northern Taiwan in the $\mathbf{2 0 1 5 - 1 6}$ season. Maximum likelihood (ML) tree of HA gene

530 coding sequences from $73 \mathrm{H} 1 \mathrm{~N} 1 \mathrm{pdm}$ viruses of the surveillance was generated using

531 RAxML under GTRGAMMA model with 1000 bootstrap replicates. Their time scales

532 based on sample collection dates, noted in each sample, were calibrated using

533 coalescent model in TreeTime after building ML tree. Reference sequences are

$534 \mathrm{H} 1 \mathrm{~N} 1 \mathrm{pdm}$ vaccine strains, A/California/07/2009 and A/Michigan/45/2015. Tips were

535 colored by location of sample collection. Samples from Baltimore were colored in brown;

536 samples from northern Taiwan were colored in green. Most samples were grouped into

537 two clusters, 6B.1 (in red) and 6B.2 (in blue). Specific amino acid mutations defining

538 major branches of 6B.1 and 6B.2 were indicated on the side of branch.

Figure 2. Four distinct genetic subgroups of H1N1pdm were identified in

Baltimore and northern Taiwan in the $\mathbf{2 0 1 5 - 1 6}$ season. Maximum likelihood (ML)

542 trees of concatenated gene segments of $73 \mathrm{H} 1 \mathrm{~N} 1 \mathrm{pdm}$ viruses of the surveillance were

543 generated using RAxML under GTRGAMMA model with 1000 bootstrap replicates.

544 Their time scales based on sample collection dates, noted in each sample, were

545 calibrated using coalescent model in TreeTime after building ML trees. Reference

546 sequences are H1N1pdm vaccine strains, A/California/07/2009 and

547 A/Michigan/45/2015. Tips were colored by location of sample collection. Samples from

548 Baltimore were colored in brown; samples from northern Taiwan were colored in green.

549 A schematic representation of clade/mutation of each gene segment based on their 
550 individual gene tree branches to separate annotated clades (1, 6B, 6B.1, or 6B.2) and

551 amino acid mutations (see Figure S1) was annotated and visualized using the ggtree $R$

552 package. Mutations (Mut) in NA, M, NS, and HA segments were identified to be able to

553 divide majority of viruses into four distinct genetic subgroups, 6B.1 parental, 6B.1

554 variant, $6 \mathrm{~B} .2$ variant-1 and $6 \mathrm{~B} .2$ variant- 2 .

555

556 Table 1. Univariate analysis of patients infected with H1N1pdm 6B.1 and 6B.2

557 viruses of Baltimore and northern Taiwan in the 2015-16 season

558

Table 2. Logistic regression analysis of pneumonia in patients infected with

H1N1pdm 6B.1 and 6B.2 viruses of Baltimore and northern Taiwan in the 2015-16

season

Table 3. Univariate analysis of patients infected with H1N1pdm 6B.1 parental and

6B.1 variant viruses of Baltimore and northern Taiwan in the 2015-16 season

Table 4. Univariate analysis of patients infected with H1N1pdm 6B.2 variant-1 and

6B.2 variant-2 viruses of Baltimore and northern Taiwan in the 2015-16 season

Supplemental Figure 1. All other segments than HA of H1N1pdm viruses in the

570

2015-16 season were differentially evolving toward either the clade 6B.1 or 6B.2.

571 Phylogenetic trees of the PB2, PB1, PA, PA-X, NP, NA, M1, M2, NS1 and NS2 coding

572 sequences (subfigures from $\mathrm{A}$ to $\mathrm{J}$, respectively) of $73 \mathrm{H} 1 \mathrm{~N} 1 \mathrm{pdm}$ viruses of the 
573 surveillance were generated using RAxML under GTRGAMMA model with 1000

574 bootstrap replicates and visualized using the ggtree $\mathrm{R}$ package. Branch tips were

575 colored by HA clades, and amino acid mutations on branch were shown. Reference

576 sequences of $\mathrm{H} 1 \mathrm{~N} 1 \mathrm{pdm}$ vaccine strains, $\mathrm{A} /$ California/07/2009 (clade 1), and

577 A/Michigan/45/2015 (clade 6B.1) were included. NP coding sequence numbering begins

578 from upstream start codon.

579

580 Supplemental Table 1. GenBank accession numbers of each segment of 73

581 H1N1pdm strains in this study.

Supplemental Table 2. Amino acid differences (H1 numbering) of HA gene in 6B.1 and 6B.2 viruses of Baltimore and northern Taiwan in the 2015-16 season

Supplemental Table 3. Amino acid differences of NA gene in 6B.1 and 6B.2 
Figure 1

Clade

6B. 1

6B.2

Location

- Baltimore

- Northern Taiwan

- Reference

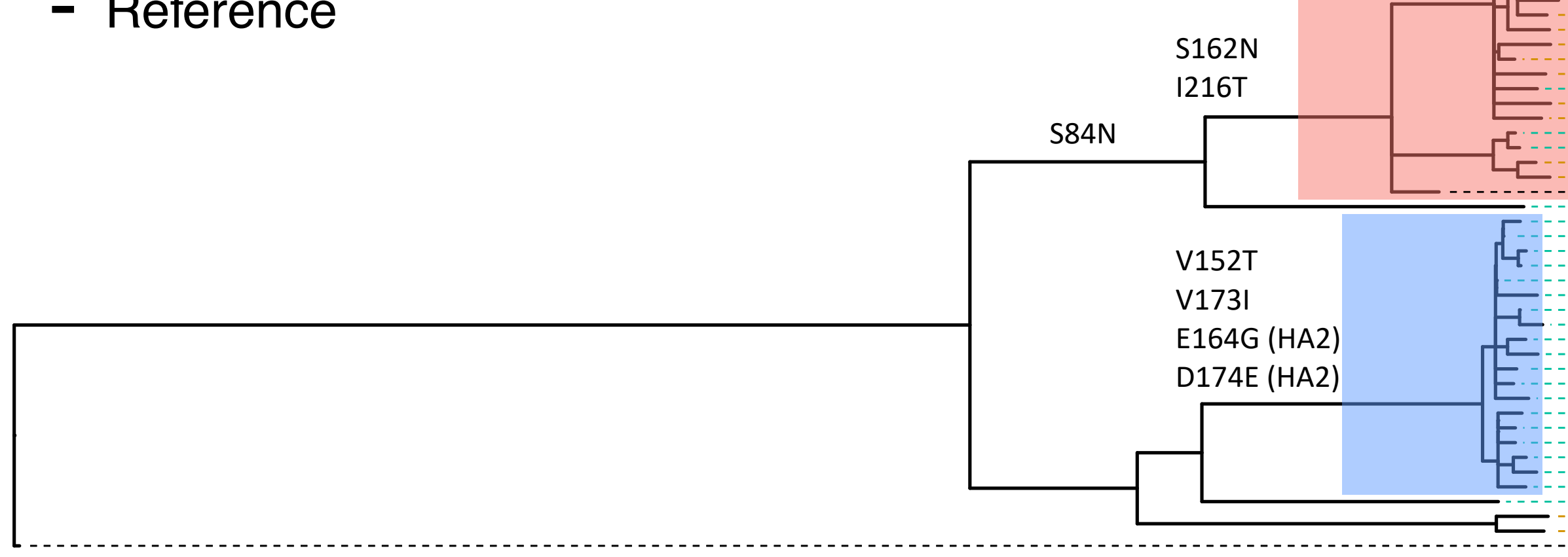




\section{Figure 2}

\section{Clade/Mutation}

1

6B

6B.1

$6 \mathrm{~B} .2$

NA-F74L

NS2-G22V

NA-S52R,G96S

M1-S30N,M2-Y52C

HA-A261S

\section{Location}

- Baltimore

- Northern Taiwan

- Reference

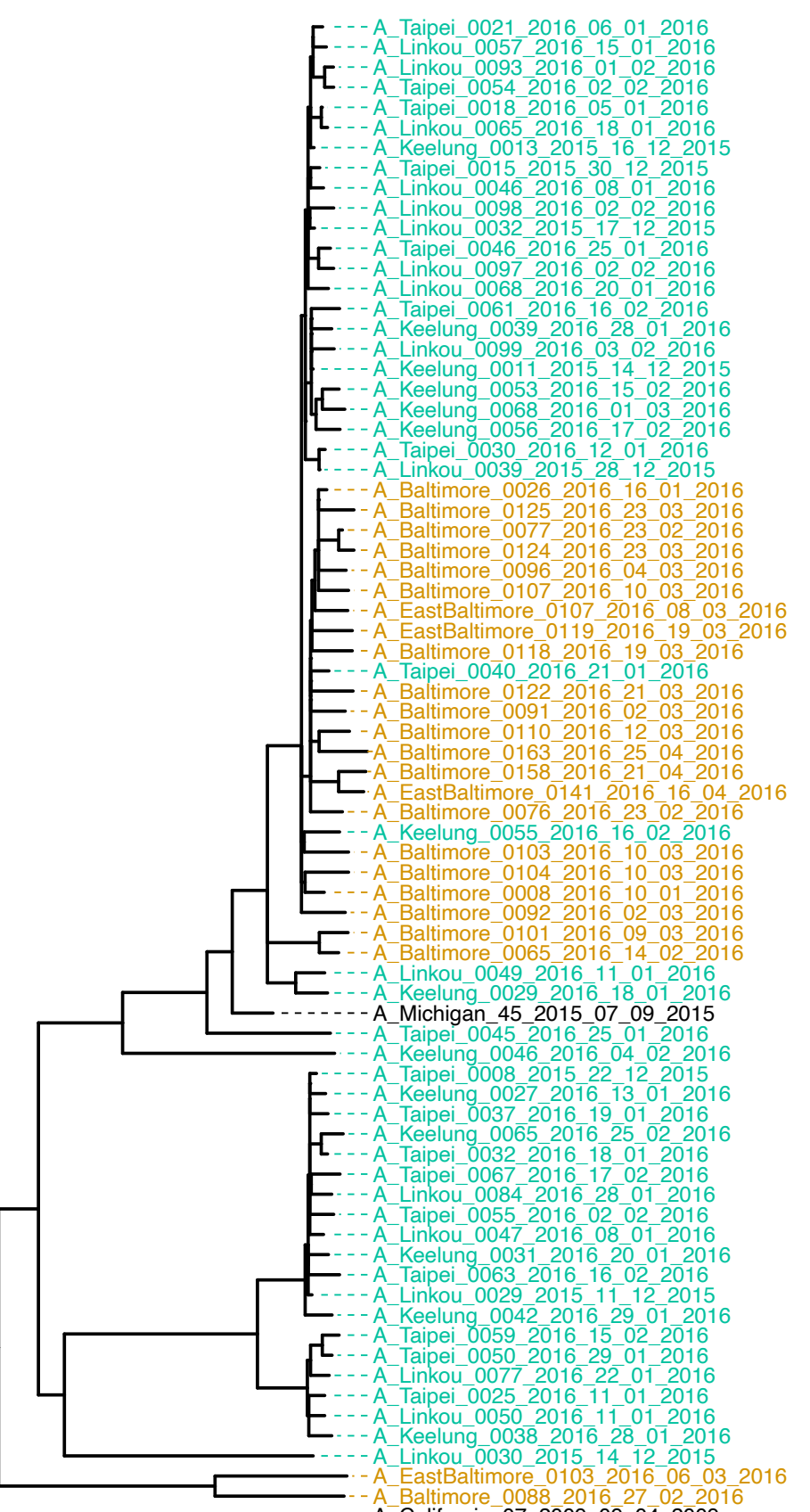


Table 1. Univariate analysis of patients infected with H1N1pdm 6B.1 and 6B.2 viruses of Baltimore and northern Taiwan in the 2015-16 season

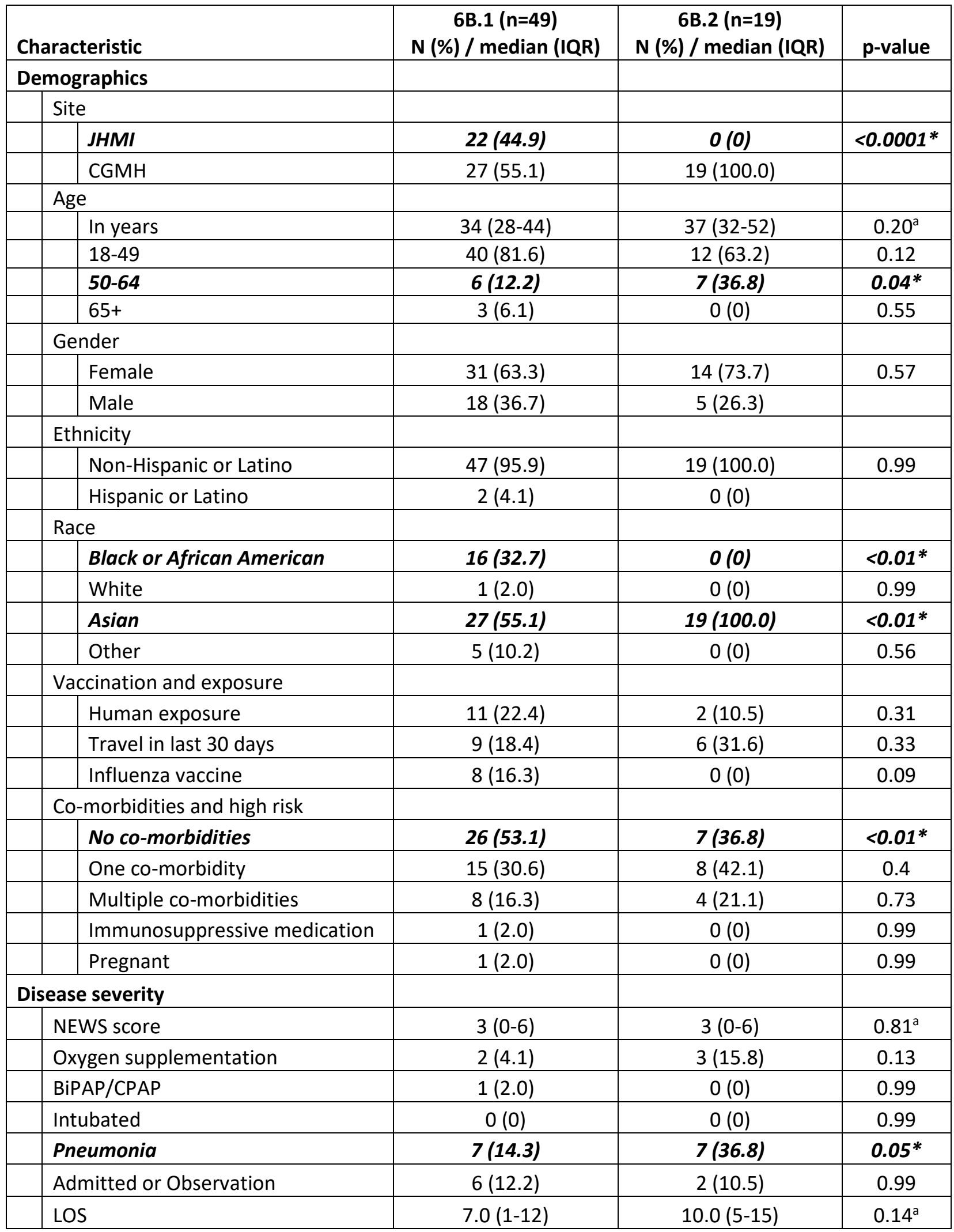




\begin{tabular}{|l|l|c|c|c|}
\hline & ICU & $0(0)$ & $0(0)$ & 0.99 \\
\hline & Death & $0(0)$ & $0(0)$ & 0.99 \\
\hline & Follow up due to sequala & $3(6.1)$ & $1(5.3)$ & 0.99 \\
\hline & Viral co-infection & $0(0)$ & $0(0)$ & 0.99 \\
\hline Symptoms & $47(95.9)$ & & \\
\hline & fever & $48(98.0)$ & $19(100.0)$ & 0.99 \\
\hline & cough & $32(65.3)$ & $19(100.0)$ & 0.99 \\
\hline & sputum & $17(34.7)$ & $14(73.7)$ & 0.77 \\
\hline & increased sputum & $35(71.4)$ & $6(31.6)$ & 0.78 \\
\hline & shortness of breath & $16(32.7)$ & $10(52.6)$ & 0.16 \\
\hline & wheezing & $40(81.6)$ & $10(52.6)$ & 0.16 \\
\hline & headache & $39(79.6)$ & $18(94.7)$ & 0.26 \\
\hline & sore throat & $43(87.8)$ & $15(78.9)$ & 0.99 \\
\hline & body aches & $\mathbf{3 7}(75.5)$ & $19(100.0)$ & 0.17 \\
\hline & runny nose & $13(26.5)$ & $19(100.0)$ & $\mathbf{0 . 0 2}$ \\
\hline & sinus pain & $43(87.8)$ & $18(94.7)$ & 0.091 \\
\hline & fatigue & $30(61.2)$ & $8(42.1)$ & 0.66 \\
\hline & chest pain & $38(77.6)$ & $16(84.2)$ & 0.18 \\
\hline & chills & $36(73.5)$ & $16(84.2)$ & 0.74 \\
\hline loss of appetite & $28(57.1)$ & $12(63.2)$ & 0.53 \\
\hline & nausea/vomiting & $\mathbf{1 0 ( 2 0 . 4 )}$ & $\mathbf{9 ( 4 7 . 4 )}$ & $\mathbf{0}$ \\
\hline & diarrhea & $11(22.4)$ & $2(10.5)$ & 0.79 \\
\hline & conjunctivitis & & \\
\hline
\end{tabular}

Statistical analysis was done using R software.

Categorical variables were compared using Chi-square or Fisher's exact tests.

${ }^{\text {a }}$ Continuous variables were compared with rank-sum test.

* Statistical significance was set at $p<0.05$. 
Table 2. Logistic regression analysis of pneumonia in patients infected with H1N1pdm 6B.1 and 6B.2 viruses of Baltimore and northern Taiwan in the 2015-16 season

\begin{tabular}{|l|l|l|l|l|l|l|l|}
\hline & & \multicolumn{3}{|c|}{ Unadjusted } & \multicolumn{3}{c|}{ IPTW** } \\
\hline Clade & Pneumonia & Odds Ratio & $\mathbf{9 5 \%}$ Cl & p-value & Odds Ratio & $\mathbf{9 5 \%}$ Cl & p-value \\
\hline 6B.1 & $14.3 \%(7 / 49)$ & 1 & - & - & 1 & - & - \\
\hline 6B.2 & $36.8 \%(7 / 19)$ & 3.500 & $1.629-5.372$ & $0.046^{*}$ & 3.261 & $1.696-4.826$ & $0.008^{*}$ \\
\hline
\end{tabular}

${ }^{*} \mathrm{p}<0.05$, logistic regression analysis.

** IPTW (inverse probability of treatment weighting) results were done by propensity score weighting with recursive partitioning algorithm (area under the ROC curve: 0.798 ) for age, gender, comorbidities, obesity, vaccine status, human exposure and travel history, then followed by logistic regression analysis. 
Table 3. Univariate analysis of patients infected with H1N1pdm 6B.1 parental and 6B.1 variant viruses of Baltimore and northern Taiwan in the 2015-16 season

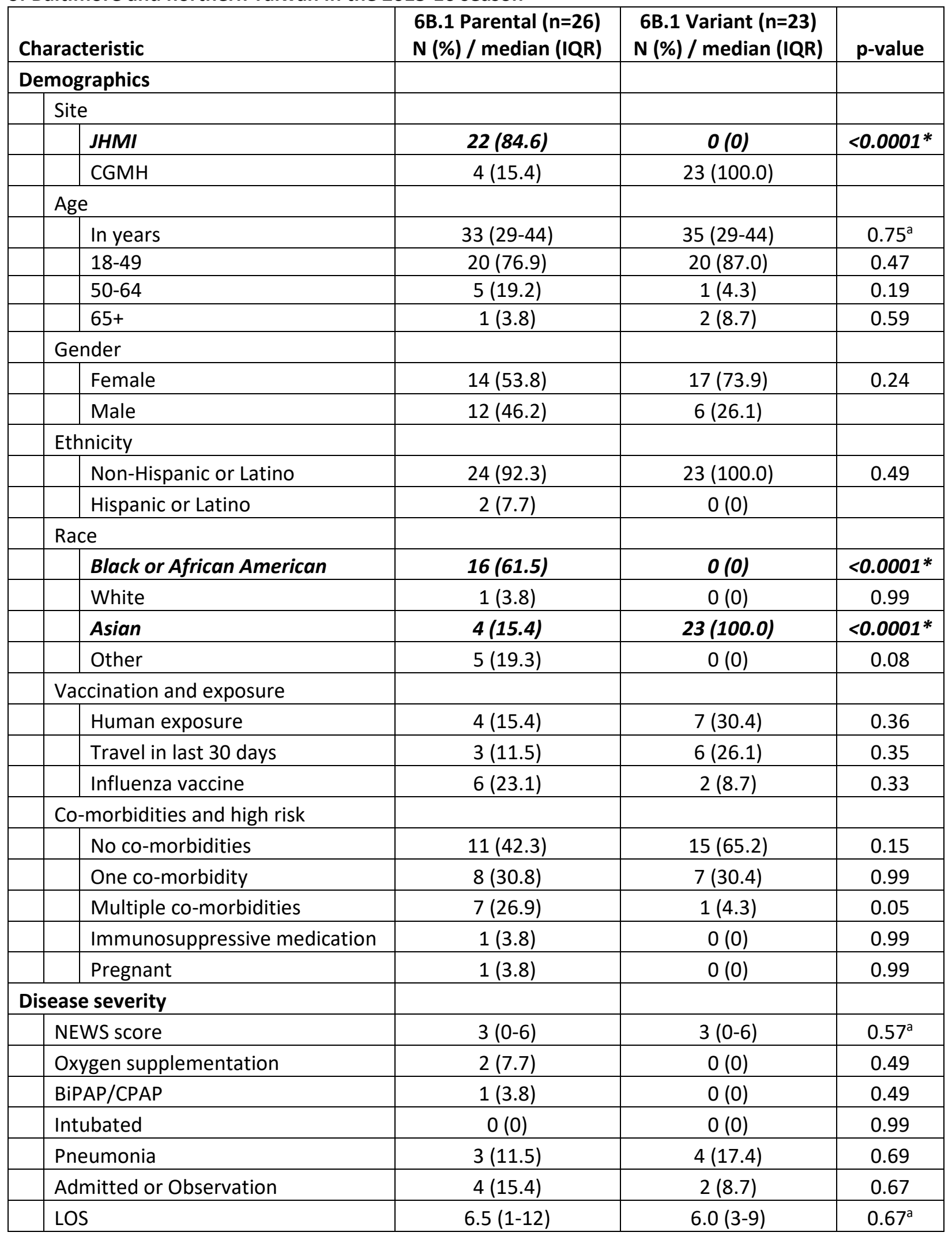




\begin{tabular}{|c|c|c|c|}
\hline ICU & $0(0)$ & $0(0)$ & 0.99 \\
\hline Death & $0(0)$ & $0(0)$ & 0.99 \\
\hline Follow up due to sequala & $2(7.7)$ & $1(4.3)$ & 0.99 \\
\hline Viral co-infection & $0(0)$ & $0(0)$ & 0.99 \\
\hline \multicolumn{4}{|l|}{ Symptoms } \\
\hline fever & $24(92.3)$ & $23(100.0)$ & 0.49 \\
\hline cough & $25(96.2)$ & $23(100.0)$ & 0.99 \\
\hline sputum & $15(57.7)$ & $17(73.9)$ & 0.37 \\
\hline increased sputum & $10(38.5)$ & $7(30.4)$ & 0.77 \\
\hline shortness of breath & $20(76.9)$ & $15(65.2)$ & 0.53 \\
\hline wheezing & $12(46.2)$ & $4(17.4)$ & $0.04^{*}$ \\
\hline headache & $23(88.5)$ & $17(73.9)$ & 0.27 \\
\hline sore throat & $19(73.1)$ & $20(87.0)$ & 0.3 \\
\hline body aches & $24(92.3)$ & $19(82.6)$ & 0.4 \\
\hline runny nose & $17(65.4)$ & $20(87.0)$ & 0.1 \\
\hline sinus pain & $11(42.3)$ & $2(8.7)$ & $0.01 *$ \\
\hline fatigue & $22(84.6)$ & $21(91.3)$ & 0.67 \\
\hline chest pain & $19(73.1)$ & $11(47.8)$ & 0.08 \\
\hline chills & $21(80.8)$ & $17(73.9)$ & 0.73 \\
\hline loss of appetite & $20(76.9)$ & $16(69.6)$ & 0.75 \\
\hline nausea/vomiting & $19(73.1)$ & 9 (39.1) & $0.02 *$ \\
\hline diarrhea & 7 (26.9) & $3(13.0)$ & 0.3 \\
\hline conjunctivitis & $8(30.8)$ & $3(13.0)$ & 0.18 \\
\hline
\end{tabular}

Statistical analysis was done using R software.

Categorical variables were compared using Chi-square or Fisher's exact tests.

${ }^{a}$ Continuous variables were compared with rank-sum test.

* Statistical significance was set at $p<0.05$. 
Table 4. Univariate analysis of patients infected with H1N1pdm 6B.2 variant-1 and 6B.2 variant-2 viruses of Baltimore and northern Taiwan in the 2015-16 season

\begin{tabular}{|c|c|c|c|}
\hline Characteristic & $\begin{array}{l}\text { 6B.2 Variant-1 ( } n=6) \\
N(\%) \text { / median (IQR) }\end{array}$ & $\begin{array}{l}6 \mathrm{~B} .2 \text { Variant-2 ( } \mathrm{n}=13 \text { ) } \\
\mathrm{N}(\%) \text { / median (IQR) }\end{array}$ & p-value \\
\hline \multicolumn{4}{|l|}{ Demographics } \\
\hline Site & & & \\
\hline JHMI & $0(0)$ & $0(0)$ & 0.99 \\
\hline $\mathrm{CGMH}$ & $6(100.0)$ & $13(100.0)$ & \\
\hline Age & & & \\
\hline In years & $34(29-48)$ & $44(34-54)$ & $0.20^{\mathrm{a}}$ \\
\hline $18-49$ & $4(66.7)$ & $8(61.5)$ & 0.99 \\
\hline $50-64$ & $2(33.3)$ & $5(38.5)$ & 0.99 \\
\hline $65+$ & $0(0)$ & $0(0)$ & 0.99 \\
\hline Gender & & & \\
\hline Female & $3(50.0)$ & $11(84.6)$ & 0.26 \\
\hline Male & $3(50.0)$ & $2(15.4)$ & \\
\hline Ethnicity & & & \\
\hline Non-Hispanic or Latino & $6(100.0)$ & $13(100.0)$ & 0.99 \\
\hline Hispanic or Latino & $0(0)$ & $0(0)$ & \\
\hline Race & & & \\
\hline Black or African American & $0(0)$ & $0(0)$ & 0.99 \\
\hline White & $0(0)$ & $0(0)$ & 0.99 \\
\hline Asian & $6(100.0)$ & $13(100.0)$ & 0.99 \\
\hline Other & $0(0)$ & $0(0)$ & 0.99 \\
\hline Vaccination and exposure & & & \\
\hline Human exposure & $0(0)$ & $2(15.4)$ & 0.83 \\
\hline Travel in last 30 days & $3(50.0)$ & $3(23.1)$ & 0.32 \\
\hline Influenza vaccine & $0(0)$ & $0(0)$ & 0.99 \\
\hline Co-morbidities and high risk & & & \\
\hline No co-morbidities & $3(50.0)$ & $4(30.8)$ & 0.77 \\
\hline One co-morbidity & $2(33.3)$ & $6(46.2)$ & 0.99 \\
\hline Multiple co-morbidities & $1(16.7)$ & $3(23.1)$ & 0.99 \\
\hline Immunosuppressive medication & $0(0)$ & $0(0)$ & 0.99 \\
\hline Pregnant & $0(0)$ & $0(0)$ & 0.99 \\
\hline Disease severity & & & \\
\hline NEWS score & $4(2-6)$ & $3(0-5)$ & $0.54^{\mathrm{a}}$ \\
\hline Oxygen supplementation & $0(0)$ & $3(23.1)$ & 0.52 \\
\hline BiPAP/CPAP & $0(0)$ & $0(0)$ & 0.99 \\
\hline Intubated & $0(0)$ & $0(0)$ & 0.99 \\
\hline Pneumonia & $2(33.3)$ & $5(38.5)$ & 0.99 \\
\hline Admitted or Observation & $0(0)$ & $2(15.4)$ & 0.99 \\
\hline LOS & $0(0-0)$ & $10.0(5-15)$ & $<0.01 *$ \\
\hline
\end{tabular}




\begin{tabular}{|c|c|c|c|}
\hline ICU & $0(0)$ & $0(0)$ & 0.99 \\
\hline Death & $0(0)$ & $0(0)$ & 0.99 \\
\hline Follow up due to sequala & $0(0)$ & $1(7.7)$ & 0.99 \\
\hline Viral co-infection & $0(0)$ & $0(0)$ & 0.99 \\
\hline \multicolumn{4}{|l|}{ Symptoms } \\
\hline fever & $6(100.0)$ & $13(100.0)$ & 0.99 \\
\hline cough & $6(100.0)$ & $13(100.0)$ & 0.99 \\
\hline sputum & $5(83.3)$ & $9(69.2)$ & 0.99 \\
\hline increased sputum & $3(50.0)$ & $3(23.1)$ & 0.32 \\
\hline shortness of breath & $2(33.3)$ & $8(61.5)$ & 0.35 \\
\hline wheezing & $2(33.3)$ & $8(61.5)$ & 0.35 \\
\hline headache & $6(100.0)$ & $12(92.3)$ & 0.99 \\
\hline sore throat & $6(100.0)$ & $9(69.2)$ & 0.26 \\
\hline body aches & $6(100.0)$ & $13(100.0)$ & 0.99 \\
\hline runny nose & $6(100.0)$ & $13(100.0)$ & 0.99 \\
\hline sinus pain & $0(0)$ & $1(7.7)$ & 0.99 \\
\hline fatigue & $6(100.0)$ & $12(92.3)$ & 0.99 \\
\hline chest pain & $2(33.3)$ & $6(46.2)$ & 0.99 \\
\hline chills & $5(83.3)$ & $11(84.6)$ & 0.99 \\
\hline loss of appetite & $6(100.0)$ & $10(76.9)$ & 0.52 \\
\hline nausea/vomiting & $3(50.0)$ & $9(69.2)$ & 0.62 \\
\hline diarrhea & 4 (66.7) & $5(38.5)$ & 0.35 \\
\hline conjunctivitis & $0(0)$ & $2(15.4)$ & 0.99 \\
\hline
\end{tabular}

Statistical analysis was done using R software.

Categorical variables were compared using Chi-square or Fisher's exact tests.

${ }^{\text {a }}$ Continuous variables were compared with rank-sum test.

* Statistical significance was set at $p<0.05$. 
Supplemental Table 1. GenBank accession numbers of each segment of $73 \mathrm{H} 1 \mathrm{N1pdm}$ strains in this study.

\begin{tabular}{|c|c|c|c|c|c|c|c|c|c|}
\hline Strain name & HA clade & PB2 & PB1 & PA & HA & NP & NA & M & NS \\
\hline A/Baltimore/0008/2016 & 6B.1 & KY487410 & KY487672 & KY487343 & KY487698 & KY487491 & KY487604 & KY487576 & KY487127 \\
\hline A/Baltimore/0026/2016 & $6 \mathrm{~B} .1$ & KY949909 & KY949764 & KY950028 & KY950126 & KY949939 & KY949753 & KY950197 & KY949800 \\
\hline A/Baltimore/0065/2016 & $6 \mathrm{~B} .1$ & KY950125 & KY949852 & KY950143 & KY950052 & KY949710 & KY949880 & KY950094 & KY950041 \\
\hline A/Baltimore/0076/2016 & $6 \mathrm{~B} .1$ & KY487315 & KY487375 & KY487419 & KY487689 & KY487608 & KY487361 & KY487616 & KY487333 \\
\hline A/Baltimore/0077/2016 & 6B.1 & KY949732 & KY949997 & KY949930 & KY950108 & KY949912 & KY949844 & KY950209 & KY950085 \\
\hline A/Baltimore/0088/2016 & $6 \mathrm{~B}$ & KY487517 & KY487300 & KY615534 & KY487140 & KY487313 & KY487269 & KY487124 & KY487551 \\
\hline A/Baltimore/0091/2016 & 6B.1 & KY487652 & KY487289 & KY487640 & KY615553 & KY615415 & KY487668 & KY487464 & KY487393 \\
\hline A/Baltimore/0092/2016 & $6 \mathrm{~B} .1$ & KY487138 & KY487194 & KY615404 & KY487543 & KY487486 & KY487463 & KY487409 & KY487653 \\
\hline A/Baltimore/0096/2016 & $6 \mathrm{~B} .1$ & KY487098 & KY615465 & KY487452 & KY615388 & KY487164 & KY487401 & KY487428 & KY487191 \\
\hline A/Baltimore/0101/2016 & 6B.1 & KY487518 & KY487065 & KY487522 & KY487503 & KY487259 & KY615395 & KY487263 & KY487762 \\
\hline A/Baltimore/0103/2016 & 6B.1 & KY487208 & KY487397 & KY487290 & KY487506 & KY487341 & KY487256 & KY487384 & KY487201 \\
\hline A/Baltimore/0104/2016 & 6B.1 & 87282 & 7502 & KY487344 & KY487646 & & KY487475 & KY487605 & KY487212 \\
\hline A/Baltimore/0107/2016 & 6B.1 & KY487556 & KY487562 & KY487722 & KY487472 & KY487183 & KY487084 & KY487451 & KY487439 \\
\hline A/Baltimore/0110/2016 & 6B.1 & KY487731 & KY615501 & KY487168 & KY615408 & KY487185 & KY615483 & KY487645 & KY487752 \\
\hline A/Baltimore/0118/2016 & $6 \mathrm{~B} .1$ & KY615383 & KY615428 & KY615505 & KY615604 & KY615376 & KY615516 & KY487552 & KY615550 \\
\hline A/Baltimore/0122/2016 & 6B.1 & KY615526 & KY615507 & KY487237 & KY615559 & KY615489 & KY615491 & KY487461 & KY615412 \\
\hline A/Baltimore/0124/2016 & 6B.1 & KY487667 & KY487566 & KY487101 & KY487575 & KY487158 & KY487434 & KY615563 & KY487666 \\
\hline A/Baltimore/0125/2016 & $6 \mathrm{~B} .1$ & KY487348 & KY487415 & KY487427 & KY487453 & KY487458 & KY487175 & KY487215 & KY487497 \\
\hline A/Baltimore/0158/2016 & 6B.1 & KY487156 & KY615599 & KY487293 & KY487430 & KY615592 & KY487539 & KY487525 & KY487281 \\
\hline A/Baltimore/0163/2016 & $6 \mathrm{~B} .1$ & KY487433 & KY487770 & KY487188 & KY487592 & KY487572 & KY487523 & KY615557 & KY487524 \\
\hline A/EastBaltimore/0103/2016 & $6 \mathrm{~B}$ & KY487534 & KY487611 & KY487704 & KY487656 & KY487615 & KY487392 & KY487465 & KY487599 \\
\hline A/EastBaltimore/0107/2016 & 6B.1 & KY487621 & KY487721 & KY487292 & KY487123 & KY487223 & KY487136 & KY487240 & KY487116 \\
\hline A/EastBaltimore/0119/2016 & $6 \mathrm{~B} .1$ & KY487252 & KY487758 & KY487325 & KY615447 & KY487381 & KY487391 & KY487596 & KY487498 \\
\hline A/EastBaltimore/0141/2016 & 6B.1 & KY615562 & KY615541 & KY615512 & KY487527 & KY487701 & KY487628 & KY487529 & KY487696 \\
\hline A/Linkou/0029/2015 & $6 \mathrm{~B} .2$ & KY949707 & KY950139 & KY949697 & KY950184 & KY950083 & KY949706 & KY950178 & KY950073 \\
\hline A/Linkou/0030/2015 & $6 \mathrm{~B}$ & KY949913 & KY950117 & KY950138 & KY949802 & KY949767 & KY949760 & KY949954 & KY949636 \\
\hline A/Linkou/0032/2015 & $6 \mathrm{~B} .1$ & KY949925 & KY949664 & KY950068 & KY949823 & KY950112 & KY949903 & KY949646 & KY950070 \\
\hline
\end{tabular}




\begin{tabular}{|c|c|c|c|c|c|c|c|c|c|}
\hline A/Linkou/0039/2015 & 6B.1 & KY949951 & KY949669 & KY949719 & KY949680 & KY949722 & KY949743 & KY949988 & KY950158 \\
\hline A/Linkou/0046/2016 & $6 B .1$ & KY949658 & KY950088 & KY949714 & KY949679 & KY949884 & KY949888 & KY949993 & KY950131 \\
\hline A/Linkou/0047/2016 & $6 B .2$ & KY949962 & KY950065 & KY949782 & KY949975 & KY950049 & KY949999 & KY949696 & KY949731 \\
\hline A/Linkou/0049/2016 & $6 B .1$ & KY950066 & KY950082 & KY950071 & KY950223 & KY949703 & KY949738 & KY949901 & KY950106 \\
\hline A/Linkou/0050/2016 & $6 B .2$ & KY949917 & KY949704 & KY949660 & KY949736 & KY950061 & KY950170 & KY949787 & KY950194 \\
\hline A/Linkou/0057/2016 & 6B.1 & KY487199 & KY615531 & KY487496 & KY615390 & KY615380 & KY487310 & KY487581 & KY487584 \\
\hline A/Linkou/0065/2016 & $6 B .1$ & KY487225 & KY487445 & KY487071 & KY487702 & KY487404 & KY487488 & KY487321 & KY487373 \\
\hline A/Linkou/0068/2016 & 6B.1 & KY487554 & KY487719 & KY487387 & KY615570 & KY487505 & KY615429 & KY487610 & KY487555 \\
\hline A/Linkou/0077/2016 & $6 B .2$ & KY487278 & KY615572 & KY487598 & KY487687 & KY487372 & KY487383 & KY487283 & KY487087 \\
\hline A/Linkou/0084/2016 & $6 B .2$ & KY487285 & KY615373 & KY487481 & KY487767 & KY487487 & KY487099 & KY487376 & KY487500 \\
\hline A/Linkou/0093/2016 & $6 B .1$ & KY487658 & KY487319 & KY487260 & KY487613 & KY487717 & KY487623 & KY487380 & KY487067 \\
\hline A/Linkou/0097/2016 & 6B.1 & KY487417 & KY615513 & KY615499 & KY615425 & KY615535 & KY487639 & KY487328 & KY487213 \\
\hline A/Linkou/0098/2016 & 6B.1 & KY487303 & KY615539 & KY487195 & KY615441 & KY487327 & KY487137 & KY487294 & KY487720 \\
\hline A/Linkou/0099/2016 & 6B.1 & KY487476 & KY487764 & KY487131 & KY487353 & KY487597 & KY487197 & KY487068 & KY487072 \\
\hline A/Taipei/0008/2015 & $6 B .2$ & KY487634 & KY487510 & KY487631 & KY487478 & KY487715 & KY487143 & KY487364 & KY487755 \\
\hline A/Taipei/0015/2015 & 6B.1 & KY487198 & KY487557 & KY487423 & KY487273 & KY615585 & KY487485 & KY487507 & KY487200 \\
\hline A/Taipei/0018/2016 & 6B.1 & KY487754 & KY487607 & KY487690 & KY487725 & KY487167 & KY487501 & KY487692 & KY487378 \\
\hline A/Taipei/0021/2016 & 6B.1 & KY487085 & KY487345 & KY487106 & KY487147 & KY615436 & KY487459 & KY487339 & KY487211 \\
\hline A/Taipei/0025/2016 & $6 B .2$ & KY487148 & KY487326 & KY487145 & KY487209 & KY487275 & KY487484 & KY487119 & KY487362 \\
\hline A/Taipei/0030/2016 & 6B.1 & KY615580 & KY487726 & KY487079 & KY487336 & KY487277 & KY487553 & KY487489 & KY487386 \\
\hline A/Taipei/0032/2016 & $6 B .2$ & KY487304 & KY487371 & KY487083 & KY487330 & KY487239 & KY487718 & KY487511 & KY487663 \\
\hline A/Taipei/0037/2016 & $6 B .2$ & KY487264 & KY487441 & KY487738 & KY487649 & KY487232 & KY615564 & KY487243 & KY487349 \\
\hline A/Taipei/0040/2016 & $6 \mathrm{~B} .1$ & KY487411 & KY487538 & KY615496 & KY487744 & KY487241 & KY487229 & KY487089 & KY487257 \\
\hline A/Taipei/0045/2016 & $6 \mathrm{~B}$ & KY487296 & KY487456 & KY487367 & KY487602 & KY487178 & KY487714 & KY487426 & KY487565 \\
\hline A/Taipei/0046/2016 & 6B.1 & KY487740 & KY487531 & KY487560 & KY487436 & KY487504 & KY487170 & KY487734 & KY487635 \\
\hline A/Taipei/0050/2016 & $6 B .2$ & KY487526 & KY487474 & KY487110 & KY487591 & KY487312 & KY487102 & KY487746 & KY487111 \\
\hline A/Taipei/0054/2016 & 6B.1 & KY487736 & KY487169 & KY487299 & KY487403 & KY487184 & KY487247 & KY487449 & KY487258 \\
\hline A/Taipei/0055/2016 & $6 \mathrm{~B} .2$ & KY487595 & KY487249 & KY487218 & KY487233 & KY487660 & KY487733 & KY487712 & KY487528 \\
\hline A/Taipei/0059/2016 & $6 \mathrm{~B} .2$ & KY487320 & KY487118 & KY615435 & KY615586 & KY615461 & KY487324 & KY487648 & KY487291 \\
\hline A/Taipei/0061/2016 & 6B.1 & KY487379 & KY487546 & KY487732 & KY487248 & KY487224 & KY487350 & KY487636 & KY487360 \\
\hline
\end{tabular}




\begin{tabular}{|c|c|c|c|c|c|c|c|c|c|}
\hline A/Taipei/0063/2016 & $6 \mathrm{~B} .2$ & KY487629 & KY487090 & KY615394 & KY487365 & KY487571 & KY487230 & KY487516 & KY487174 \\
\hline A/Taipei/0067/2016 & $6 \mathrm{~B} .2$ & KY487311 & KY615407 & KY487470 & KY487709 & KY487388 & KY487675 & KY487255 & KY487226 \\
\hline A/Keelung/0011/2015 & $6 \mathrm{~B} .1$ & KY487600 & KY487113 & KY487166 & KY487251 & KY487574 & KY487437 & KY487699 & KY487593 \\
\hline A/Keelung/0013/2015 & 6B.1 & KY950069 & KY949665 & KY949995 & KY949643 & KY950031 & KY950092 & KY949803 & KY950115 \\
\hline A/Keelung/0027/2016 & $6 \mathrm{~B} .2$ & KY487512 & KY487713 & KY487086 & KY487340 & KY487676 & KY487231 & KY487134 & KY487418 \\
\hline A/Keelung/0029/2016 & $6 \mathrm{~B} .1$ & KY615478 & KY487334 & KY615481 & KY487499 & KY487769 & KY615400 & KY487400 & KY487314 \\
\hline A/Keelung/0031/2016 & $6 \mathrm{~B} .2$ & KY487363 & KY487573 & KY615579 & KY615480 & KY487589 & KY487204 & KY487276 & KY487444 \\
\hline A/Keelung/0038/2016 & $6 \mathrm{~B} .2$ & KY487483 & KY487739 & KY487614 & KY487655 & KY487268 & KY487466 & KY487091 & KY487129 \\
\hline A/Keelung/0039/2016 & $6 \mathrm{~B} .1$ & KY949952 & KY949976 & KY950181 & KY949887 & KY949948 & 9872 & KY949898 & KY949946 \\
\hline A/Keelung/0042/2016 & $6 \mathrm{~B} .2$ & KY487244 & KY487186 & KY487187 & KY487695 & KY487274 & KY615567 & KY487399 & KY615457 \\
\hline A/Keelung/0046/2016 & $6 \mathrm{~B} .1$ & KY487665 & KY487761 & KY615468 & KY487149 & KY487406 & KY487271 & KY487245 & KY487217 \\
\hline A/Keelung/0053/2016 & $6 \mathrm{~B} .1$ & KY487594 & KY487609 & KY487684 & KY487727 & KY487088 & KY487181 & KY487177 & KY487748 \\
\hline A/Keelung/0055/2016 & $6 \mathrm{~B} .1$ & KY949741 & KY949863 & KY950220 & KY950148 & KY949733 & KY949761 & KY949653 & KY950042 \\
\hline A/Keelung/0056/2016 & $6 \mathrm{~B} .1$ & KY487082 & KY487495 & KY615527 & KY487617 & KY487368 & KY487416 & KY487443 & KY487165 \\
\hline A/Keelung/0065/2016 & $6 \mathrm{~B} .2$ & KY487674 & KY487216 & KY615378 & KY487219 & KY487567 & KY615459 & KY487075 & KY487579 \\
\hline A/Keelung/0068/2016 & 6B.1 & KY487114 & KY487742 & KY487180 & KY615443 & KY487374 & KY487728 & KY487105 & KY487228 \\
\hline
\end{tabular}


Supplemental Table 2. Amino acid differences (H1 numbering) of HA gene in 6B.1 and 6B.2 viruses of Baltimore and northern Taiwan in the 2015-16 season

\begin{tabular}{|c|c|c|c|c|c|c|c|c|c|c|}
\hline Vaccine/ Surveillance strains ${ }^{*}$ & $\begin{array}{l}\text { HA1- } \\
84\end{array}$ & $\begin{array}{l}\text { HA1- } \\
152\end{array}$ & $\begin{array}{l}\text { HA1- } \\
162\end{array}$ & $\begin{array}{l}\text { HA1- } \\
173\end{array}$ & $\begin{array}{l}\text { HA1- } \\
209\end{array}$ & $\begin{array}{l}\text { HA1- } \\
216\end{array}$ & $\begin{array}{l}\text { HA1- } \\
223\end{array}$ & $\begin{array}{l}\text { HA1- } \\
261\end{array}$ & $\begin{array}{l}\text { HA2- } \\
164\end{array}$ & $\begin{array}{l}\text { HA2- } \\
174\end{array}$ \\
\hline A/California/7/2009 & $\mathrm{S}$ & $\mathrm{V}$ & $\mathrm{S}$ & $\mathrm{V}$ & $T^{\&}$ & I & $R^{\&}$ & $A$ & $\mathrm{E}$ & $\mathrm{D}$ \\
\hline A/Michigan/45/2015 & $\mathrm{N}$ & $\mathrm{V}$ & $\mathrm{N}^{\mathrm{Gly}+}$ & V & $M^{\&}$ & $\mathrm{~T}$ & $\mathrm{R}^{\&}$ & A & $E$ & $\mathrm{D}$ \\
\hline 6B.1 parental $(n=26)$ & $\mathrm{N}$ & V & $\mathrm{N}^{\mathrm{Gly}+}$ & V & $\mathrm{K}$ & $\mathrm{T}$ & Q & A & $E$ & $\mathrm{D}$ \\
\hline $6 B .1$ variant $(n=23)$ & $\mathrm{N}$ & V & $\mathrm{N}^{\mathrm{Gly}+}$ & V & $\mathrm{K}$ & $\mathrm{T}$ & Q & A & $\mathrm{E}$ & $\mathrm{D}$ \\
\hline $6 B .2$ variant $-1(n=6)$ & $\mathrm{S}$ & $\mathrm{T}$ & $\mathrm{S}$ & I & $\mathrm{K}$ & 1 & Q & $\mathrm{S}$ & G & $E$ \\
\hline $6 B .2$ variant $-2(n=13)$ & $\mathrm{S}$ & $\mathrm{T}$ & $\mathrm{S}$ & 1 & $\mathrm{~K}$ & 1 & Q & $A$ & G & $E$ \\
\hline $6 B .1$ reassortant $(n=1)$ & $\mathrm{N}$ & V & $\mathrm{N}^{\mathrm{Gly}+}$ & V & K & $\mathrm{T}$ & $Q$ & $A$ & $\mathrm{E}$ & $\mathrm{D}$ \\
\hline
\end{tabular}

* Twenty-six strains of 6B.1 parental group include 22 Baltimore and 4 northern Taiwan strains. 6B.1 variant, 6B.2 variant-1 and -2, and 6B.1 reassortant strains are all isolated from northern Taiwan.

Gly+ Amino acid changes lead to gain of potential N-linked glycosylation site.

\& Mutations of clade $1 \mathrm{~A} /$ California/7/2009 vaccine X-179A or clade 6B.1 A/Michigan/45/2015 vaccine X275. The HA1 sequences of clinical isolates of A/California/7/2009 and A/Michigan/45/2015 were 209K and 223Q. 
Supplemental Table 3. Amino acid differences of NA gene in 6B.1 and 6B.2 viruses of Baltimore and northern Taiwan in the 2015-16 season

\begin{tabular}{|c|c|c|c|c|c|c|c|c|}
\hline Vaccine/ Surveillance strains ${ }^{*}$ & $\begin{array}{l}\text { NA- } \\
13\end{array}$ & $\begin{array}{l}\text { NA- } \\
\mathbf{3 4}\end{array}$ & $\begin{array}{l}\text { NA- } \\
52\end{array}$ & $\begin{array}{l}\text { NA- } \\
67\end{array}$ & $\begin{array}{l}\text { NA- } \\
74\end{array}$ & $\begin{array}{l}\text { NA- } \\
96\end{array}$ & $\begin{array}{l}\text { NA- } \\
314\end{array}$ & $\begin{array}{l}\text { NA- } \\
381\end{array}$ \\
\hline A/California/7/2009 & V & 1 & $\mathrm{~S}$ & V & $\mathrm{F}$ & G & 1 & $\mathrm{~T}$ \\
\hline A/Michigan/45/2015 & 1 & V & $\mathrm{S}$ & V & $\mathrm{F}$ & G & M & $\mathrm{T}$ \\
\hline 6B.1 parental $(n=26)$ & 1 & $\mathrm{~V}$ & $\mathrm{~S}$ & V & $\mathrm{F}$ & G & M & $\mathrm{T}$ \\
\hline $6 B .1$ variant $(n=23)$ & 1 & $\mathrm{~V}$ & $\mathrm{~S}$ & V & L & G & M & $\mathrm{T}$ \\
\hline $6 B .2$ variant $-1(n=6)$ & V & 1 & $S$ & 1 & $\mathrm{~F}$ & G & 1 & 1 \\
\hline $6 B .2$ variant $-2(n=13)$ & V & 1 & $\mathrm{R}^{\mathrm{Gly}-}$ & 1 & $\mathrm{~F}$ & $S$ & 1 & 1 \\
\hline 6B.1 reassortant $(n=1)$ & V & 1 & $\mathrm{R}^{\text {Gly- }}$ & 1 & $\mathrm{~F}$ & $S$ & 1 & 1 \\
\hline
\end{tabular}

${ }^{*}$ Twenty-six strains of 6B.1 parental group include 22 Baltimore and 4 northern Taiwan strains. 6 B.1 variant, 6 B. 2 variant-1 and -2 , and 6B.1 reassortant strains are all isolated from northern Taiwan.

Gly- Amino acid changes lead to loss of potential N-linked glycosylation site. 
Supplemental Table 4. Amino acid differences of internal genes in 6B.1 and 6B.2 viruses of Baltimore and northern Taiwan in the 2015-16 season

\begin{tabular}{|c|c|c|c|c|c|c|c|c|c|c|c|c|c|}
\hline Vaccine/ Surveillance strains ${ }^{*}$ & $\begin{array}{l}\text { PB2- } \\
299\end{array}$ & $\begin{array}{l}\text { PB2- } \\
453\end{array}$ & $\begin{array}{l}\text { PA- } \\
361\end{array}$ & $\begin{array}{l}\text { PA-X- } \\
212\end{array}$ & $\begin{array}{l}\text { NP- } \\
6^{\&}\end{array}$ & $\begin{array}{l}\text { M1- } \\
30\end{array}$ & $\begin{array}{l}\text { M1- } \\
208\end{array}$ & $\begin{array}{l}\text { M2- } \\
52\end{array}$ & $\begin{array}{l}\text { NS1- } \\
2\end{array}$ & $\begin{array}{l}\text { NS1- } \\
125\end{array}$ & $\begin{array}{l}\text { NS2- } \\
2\end{array}$ & $\begin{array}{l}\text { NS2- } \\
22\end{array}$ & $\begin{array}{l}\text { NS2- } \\
83\end{array}$ \\
\hline A/California/7/2009 & $\mathrm{R}$ & $\mathrm{S}$ & $\mathrm{K}$ & $A$ & $A$ & $\mathrm{~S}$ & $Q$ & $Y$ & $\mathrm{D}$ & $E$ & $\mathrm{D}$ & G & $M$ \\
\hline A/Michigan/45/2015 & $\mathrm{R}$ & $\mathrm{P}$ & $\mathrm{K}$ & $A$ & $A$ & $\mathrm{~S}$ & $\mathrm{~K}$ & $Y$ & $E$ & $\mathrm{D}$ & $E$ & G & 1 \\
\hline 6B.1 parental $(n=26)$ & $\mathrm{K}$ & $\mathrm{T}$ & $\mathrm{K}$ & A & A & $\mathrm{S}$ & $\mathrm{K}$ & $Y$ & $\mathrm{E}$ & $\mathrm{D}$ & $\mathrm{E}$ & G & I \\
\hline $6 B .1$ variant $(n=23)$ & $\mathrm{K}$ & $\mathrm{T}$ & $\mathrm{K}$ & $A$ & $A$ & $\mathrm{~S}$ & $\mathrm{~K}$ & $Y$ & $\mathrm{E}$ & $\mathrm{D}$ & $\mathrm{E}$ & V & I \\
\hline $6 B .2$ variant $-1(n=6)$ & $\mathrm{R}$ & $P$ & $\mathrm{R}$ & V & V & $\mathrm{S}$ & $Q$ & $Y$ & $\mathrm{D}$ & $\mathrm{E}$ & $\mathrm{D}$ & G & $\mathrm{M}$ \\
\hline $6 B .2$ variant $-2(n=13)$ & $\mathrm{R}$ & $\mathrm{P}$ & $\mathrm{R}$ & $\mathrm{V}$ & V & $\mathrm{N}$ & $Q$ & C & $\mathrm{D}$ & $\mathrm{E}$ & $\mathrm{D}$ & G & $M$ \\
\hline $6 B .1$ reassortant $(n=1)$ & $\mathrm{K}$ & $\mathrm{T}$ & $\mathrm{K}$ & A & V & $\mathrm{N}$ & $Q$ & $\mathrm{C}$ & $\mathrm{D}$ & $E$ & $\mathrm{D}$ & G & $\mathrm{M}$ \\
\hline
\end{tabular}

${ }^{*}$ Twenty-six strains of 6B.1 parental group include 22 Baltimore and 4 northern Taiwan strains. 6 B.1 variant, 6B.2 variant-1 and -2 , and 6B.1 reassortant strains are all isolated from northern Taiwan.

\& NP coding sequence numbering begins from upstream start codon. 